\title{
Epidemiology, presentation and outcome of patients with melioidosis in a tertiary care center
}

\author{
DLBPiyasiri $^{1}$, EMCorea ${ }^{2}$, SVathshalan $^{1}$, TGamage ${ }^{1}$, MCTJayasundera ${ }^{1}$, KDDSWijeweera ${ }^{1}$, \\ PMSapukotana $^{1}$, NLiyanage ${ }^{1}$, WUPriyadharshana ${ }^{1}$, WAPPriyarangani ${ }^{1}$, JVGMJayasekara ${ }^{1}$, \\ TKSSamarawickrama ${ }^{1}$, CNThewarapperuma ${ }^{1}$, IRSNanayakkara ${ }^{1}$, DIMadumali ${ }^{1}$, \\ AHWeerathunga ${ }^{1}$, LUAPriyanganie ${ }^{1}$, EHDSKumari ${ }^{1}$, RCWPathirana ${ }^{1}$, DCAVithanage ${ }^{1}$, \\ KWSMalkanthi ${ }^{1}$, HHLUdayangani ${ }^{1}$
}

\section{Introduction}

Melioidosis, caused by the Gram negative bacterium Burkholderiapseudomallei, is transmitted to humans through direct contact with contaminated soil and water. This research study aimed to describe the epidemiology, presentations and outcome of patients with melioidosis

\section{Methods}

Surveillance was carried out from 1st December 2014 to 30th June 2017 and included all culture and antibody positive patients. In the absence of a positive culture, patients with antibody levels $>40$ were included if clinical evidence of melioidosis was present. Data were collected from the patients and clinical notes.

\section{Results}

Melioidosis was diagnosed in 47 patients during this period of whom 36 (77\%) were males. The 41-60 year age group were the most affected $(n=25,53 \%)$ with only 3 paediatric ( $<10$ years) and 13 elderly (> 60 years) patients. All the patients were from the Southern Province of Sri Lanka, more than $50 \%$ giving a history of constant soil exposure and 6 had been affected by floods. .Eighteen (39\%) patients were diagnosed by isolation of the bacterium from blood, sputum, pus or urine, with or without a positive antibody test. Twenty nine patients were identified only by a positive antibody test with a compatible clinical presentation. Blood culture was positive in 15 patients. A total of $32(68 \%)$ patients had comorbidities of whom $20(42 \%)$ were diabetic. One was pregnant and one had post-partum pneumonia. Lungs were commonly affected with12 (26\%) presenting with severe pneumonia and 10 (21\%) with lung abscess. There were 4 patients with liver abscess, 4 with septic arthritis and 2 cases of endocarditis. Other presentations included deep seated abscesses, cellulitis and urinary tract infection. A focus could not be identified in 5 patients. Case fatality rate was $11 \%$. Thirty three patients completed the eradication phase with no relapses while 6 are still undergoing treatment.

\section{Discussion and Conclusions}

In this study population, melioidosis had a male predominance and affected the middle aged and elderly with comorbidities including diabetes. A high index of suspicion led to meticulous investigation and early diagnosis, especially among patients with a history of high risk exposure to soil and water.

\footnotetext{
${ }^{1}$ Teaching Hospital, Karapitiya, Sri Lanka

${ }^{2}$ Department of Microbiology, Faculty of Medicine, University of Colombo, Colombo, Sri Lanka

Address for correspondence: Dr Bhagya, Piyasiri, Teaching Hospital, Karapitiya +94 777654317

Email:bhagyapiyasiri@yahoo.co.uk https://orcid.org/0000-0002-4660-311X
} 